\title{
The Sums of Alternating Series of Odd Powers of the Reciprocals of Odd Positive Integers
}

\author{
Mohammed R. Karim \\ Department of Mathematics, Alabama A\&M University, Normal, AL, USA \\ E-mail: mohammed.karim@aamu.edu. Tel: (256) 372-4835. Fax: (256) 372-5931
}

Received April 29, 2010 / Accepted May 18, 2010

\begin{abstract}
This paper is an extension of a recent work done by the author [4] and here the sums of alternating series of odd powers (up to fifteen) of the reciprocals of odd positive integers are computed. Following this method, the sum of the series of any higher power could be calculated. In the process of computing these sums, the sums of the series of even powers of reciprocals of odd positive integers have been reestablished and enabled the author to compute the values of Riemann's zeta function for even positive integers.
\end{abstract}

Key Words: Alternating Series, Riemann Zeta Function, Sum of Series. 
To establish the claims of this paper, the author has developed the results summarized as the following lemmas, theorems and corollaries.

\section{Lemma 1.1 .}

$$
\sum_{n=0}^{\infty} \frac{\sin (2 n+1) \theta}{2 n+1}=\frac{\pi}{4}
$$

Proof: Replacing $x$ by $x^{2}$ in the geometric series

$$
\frac{1}{1-x}=1+x+x^{2}+x^{3}+x^{4}+\cdots, \quad|x|<1
$$

\section{One can write}

$$
\frac{1}{1-x^{2}}=1+x^{2}+x^{4}+x^{6}+\cdots, \quad x^{2}<1
$$

But

$$
\sum_{n=0}^{\infty} x^{2 n}=\frac{1}{1-x^{2}}
$$

Integrating both sides of (3) from 0 to $z,|z|<1$, one gets

$$
\sum_{n=0}^{\infty} \frac{z^{2 n+1}}{2 n+1}=\frac{1}{2}[\ln (1+z)-\ln (1-z)]=\frac{1}{2} \ln \left(\frac{1+z}{1-z}\right)
$$

The substitution of $z=e^{i \theta}, 0<\theta<\pi$, in (4) (by Abel's Limit Theorem [5]) gives

$$
\sum_{n=0}^{\infty} \frac{\cos (2 n+1) \theta+i \sin (2 n+1) \theta}{2 n+1}=\frac{1}{2} \ln \left(\frac{1+\cos (\theta)+i \sin (\theta)}{1-\cos (\theta)-i \sin (\theta)}\right)
$$


The argument of the logarithmic function on the right side of (5) can be simplified as

$$
\begin{aligned}
\frac{1+\cos (\theta)+i \sin (\theta)}{1-\cos (\theta)-i \sin (\theta)}= & \frac{1+\cos (\theta)+i \sin (\theta)}{1-\cos (\theta)-i \sin (\theta)} \cdot \frac{1-\cos (\theta)+i \sin (\theta)}{1-\cos (\theta)+i \sin (\theta)} \\
& =\frac{1-\cos ^{2}(\theta)-\sin ^{2}(\theta)+i\{\sin (\theta)+\cos (\theta) \sin (\theta)+\sin (\theta)-\cos (\theta) \sin (\theta)\}}{(1-\cos (\theta))^{2}+\sin ^{2}(\theta)} \\
& =\frac{\sin (\theta)}{1-\cos (\theta)} i=\frac{2 \sin (\theta / 2) \cos (\theta / 2)}{2 \sin ^{2}(\theta / 2)} i=i \cot (\theta / 2)
\end{aligned}
$$

The equation (7) provides the relation $\arg (i \cot (\theta / 2))=\frac{\pi}{2} ;$ but $\ln (z)=\ln (|z|)+i \arg (z)$ [7]; hence, one can write

$$
\frac{1}{2} \ln \left(\frac{1+z}{1-z}\right)=\frac{1}{2} \ln (\cot (\theta / 2))+i \frac{\pi}{4}
$$

Now equating the imaginary parts from (6) and (8) one can get the desired result.

$$
\sum_{n=0}^{\infty} \frac{\sin (2 n+1) \theta}{2 n+1}=\frac{\pi}{4}
$$

This relation is crucial for the present work and will be used extensively.

Theorem 1.1. For $k \geq 1$, one has

$$
\begin{aligned}
& \sum_{n=o}^{\infty} \frac{\cos (2 n+1) \theta}{(2 n+1)^{2 k}}=a_{2 k-1} \pi \theta^{2 k-1}+\sum_{i=1}^{k} a_{2 k-2 i} \pi^{2 i} \theta^{2 k-2 i} \\
& \sum_{n=0}^{\infty} \frac{\sin (2 n+1) \theta}{(2 n+1)^{2 k+1}}=b_{2 k} \pi \theta^{2 k}+\sum_{i=0}^{k} b_{2 k-2 i+1} \pi^{2 i} \theta^{2 k-2 i+1}
\end{aligned}
$$


where for $1 \leq i \leq k, a_{2 k-1}, a_{2 k-2 i}, b_{2 k}, b_{2 k-2 i+1}$ are rational numbers.

Note: The above rational numbers are not fixed constants; each of them is a function of the index $k$, and the symbol $a_{0}$, for example, will have different values for different values of $k$ ( $a_{0}=\frac{1}{8}$ for $k=1$ and $a_{0}=\frac{1}{96}$ for $k=2$ etc.).

Proof: Mathematical Induction will be used to prove the theorem. For $k=1$, first the statement of the Theorem 1.1 is true with

$$
\sum_{n=0}^{\infty} \frac{\cos (2 n+1) \theta}{(2 n+1)^{2}}=a_{1} \pi \theta+a_{0} \pi^{2}=-\frac{1}{4} \pi \theta+\frac{1}{8} \pi^{2}
$$

The rational numbers $a_{1}$ and $a_{0}$ are calculated by integrating the expression in Lemma 1.1., and by using $\theta=\pi / 2$

It is assumed that it is true for $k=r$, where $r$ is a positive integer. That is,

$$
\sum_{n=0}^{\infty} \frac{\cos (2 n+1) \theta}{(2 n+1)^{2 r}}=a_{2 r-1} \pi \theta^{2 r-1}+\sum_{i=1}^{r} a_{2 k-2 i} \pi^{2 i} \theta^{2 r-2 i}
$$

The proof would be over if one could show that the statement is true for $k=r+1$, that is,

$$
\sum_{n=0}^{\infty} \frac{\cos (2 n+1) \theta}{(2 n+1)^{2(r+1)}}=a_{2 r+1} \pi \theta^{2 r+1}+\sum_{i=1}^{r+1} a_{2 r-2 i+2} \pi^{2 i} \theta^{2 r-2 i+2} .
$$

Integration of (12) with respect to (w. r. t.) $\theta$ gives

$$
\sum_{n=0}^{\infty} \frac{\sin (2 n+1) \theta}{(2 n+1)^{2 r+1}}+C_{1}=\frac{a_{2 r-1}}{2 r} \pi \theta^{2 r}+\sum_{i=1}^{r} \frac{a_{2 r-2 i}}{(2 r-2 i+1)} \pi^{2 i} \theta^{2 r-2 i+1} .
$$


Substitution of $\theta=0$ shows that $C_{1}=0$, which turns (14) into

$$
\sum_{n=0}^{\infty} \frac{\sin (2 n+1) \theta}{(2 n+1)^{2 r+1}}=\frac{a_{2 r-1}}{2 r} \pi \theta^{2 r}+\sum_{i=1}^{r} \frac{a_{2 r-2 i}}{(2 r-2 i+1)} \pi^{2 i} \theta^{2 r-2 i+1}
$$

Integration of (15) with respect to $\theta$ produces the equation

$$
-\sum_{n=0}^{\infty} \frac{\cos (2 n+1) \theta}{(2 n+1)^{2 r+2}}+C_{2}=\frac{a_{2 r-1}}{2 r(2 r+1)} \pi \theta^{2 r+1}+\sum_{i=1}^{r} \frac{a_{2 r-2 i}}{(2 r-2 i+1)(2 r-2 i+2)} \pi^{2 i} \theta^{2 r-2 i+2}
$$

Substituting $\theta=0$ in (16), one can show that $C_{2}=\sum_{n=0}^{\infty} \frac{1}{(2 n+1)^{2(r+1)}}=a_{0} \pi^{2(r+1)}$ by repeated integration of (9).

Then (16) becomes

$$
\sum_{n=0}^{\infty} \frac{\cos (2 n+1) \theta}{(2 n+1)^{2 r+2}}=a_{0} \pi^{2(r+1)}-\frac{a_{2 r-1}}{2 r(2 r+1)} \pi \theta^{2 r+1}-\sum_{i=1}^{r} \frac{a_{2 r-2 i}}{(2 r-2 i+1)(2 r-2 i+2)} \pi^{2 i} \theta^{2 r-2 i+2}
$$

Combining the first term of the right side of (17) with the summation term and writing $a_{2 r+1}=-\frac{a_{2 r-1}}{2 r(2 r+1)}$ results

in

$$
\sum_{n=0}^{\infty} \frac{\cos (2 n+1) \theta}{(2 n+1)^{2(r+1)}}=a_{2 r+1} \pi \theta^{2 r+1}+\sum_{i=1}^{r+1} a_{2 r-2 i+2} \pi^{2 i} \theta^{2 r-2 i+2}
$$

This establishes the first part of Theorem 1.1. Following the same technique as in the proof of the first part of Theorem 1.1., one can prove the second part of it.

Corollary 1.1. For $k \geq 1$, one has

$$
\sum_{n=0}^{\infty} \frac{1}{(2 n+1)^{2 k}}=a_{0} \pi^{2 k}
$$




$$
\text { and } \sum_{n=0}^{\infty} \frac{(-1)^{n}}{(2 n+1)^{2 k+1}}=b_{2 k} \pi\left(\frac{\pi}{2}\right)^{2 k}+\sum_{i=1}^{k} b_{2 k-2 i+1} \pi^{2 i}\left(\frac{\pi}{2}\right)^{2 k-2 i+1} \text {. }
$$

One can easily prove Corollary 1.1 by substituting $\theta=0$ and $\theta=\frac{\pi}{2}$, respectively, in the first and second part of

Theorem 1.1, and using Lemma 1.1.

Using the second equation of Corollary 1.1., and Lemma 1.1., results in the following:

$$
\begin{aligned}
1-\frac{1}{3}+\frac{1}{5}-\frac{1}{7}+\cdots & =\frac{\pi}{4} . \\
1-\frac{1}{3^{3}}+\frac{1}{5^{3}}-\frac{1}{7^{3}}+\cdots & =\frac{\pi^{3}}{32} . \\
\frac{1}{1^{5}}-\frac{1}{3^{5}}+\frac{1}{5^{5}}-\frac{1}{7^{5}}+\cdots & =\frac{5}{1536} \pi^{5} . \\
\frac{1}{1^{7}}-\frac{1}{3^{7}}+\frac{1}{5^{7}}-\frac{1}{7^{7}}+\cdots & =\frac{61}{184320} \pi^{7} . \\
\frac{1}{1^{9}}-\frac{1}{3^{9}}+\frac{1}{5^{9}}-\frac{1}{7^{9}}+\cdots & =\frac{277}{8257563} \pi^{9} . \\
\frac{1}{1^{15}}-\frac{1}{3^{15}}+\frac{1}{5^{15}}-\frac{1}{7^{15}}+\cdots & =\frac{199360981}{5713316492083200} \pi^{15} \\
\frac{1}{1^{13}}-\frac{1}{3^{11}}+\frac{1}{5^{11}}-\frac{1}{7^{11}}+\cdots & =\frac{50521}{14863564800} \pi^{11} . \\
5^{13}-\frac{1}{7^{13}}+\cdots & =\frac{540553}{1569592442880} \pi^{13} .
\end{aligned}
$$


Equation (19) is known as the Leibniz Formula for $\pi[8]$ and the relation (20) was produced by Euler in 1774 [1, 3 , 6].

Continuing the above process, one can compute the sum of the series of any power of the reciprocals of odd positive integers. However, as the exponents get larger and larger, the sum gets closer and closer to 1 . One can see it from (26), where the exponent is only 15 ; the sum is approximately equal to 0.9999999303 , which is close to 1 and for this reason, the author will stop here.

As a byproduct of this work, knowing the values of the sum of the series of the even powers of the reciprocals of the odd positives integers from Corollary 1.1., one can easily compute the values of Riemann's zeta function [2] at even positive integers. To this end, one needs the following results written as lemma and corollary.

Lemma 1.2. Riemann's zeta function

$$
\zeta(n)=\sum_{i=1}^{\infty} \frac{1}{i^{n}}
$$

can be rewritten as

$$
\zeta(n)=\frac{2^{n}}{2^{n}-1} \sum_{j=0}^{\infty} \frac{1}{(2 j+1)^{n}}
$$

Proof: $\zeta(n)=\frac{1}{1^{n}}+\frac{1}{2^{n}}+\frac{1}{3^{n}}+\frac{1}{4^{n}}+\frac{1}{5^{n}}+\frac{1}{6^{n}}+\cdots$

$$
\begin{aligned}
& =\left[\frac{1}{1^{n}}+\frac{1}{3^{n}}+\frac{1}{5^{n}}+\frac{1}{7^{n}}+\cdots\right]+\left[\frac{1}{2^{n}}+\frac{1}{4^{n}}+\frac{1}{6^{n}}+\frac{1}{8^{n}}+\cdots\right] \\
& =\left[\frac{1}{1^{n}}+\frac{1}{3^{n}}+\frac{1}{5^{n}}+\frac{1}{7^{n}}+\cdots\right]+\frac{1}{2^{n}}\left[\frac{1}{1^{n}}+\frac{1}{2^{n}}+\frac{1}{3^{n}}+\frac{1}{4^{n}}+\cdots\right] \\
& =\left(1+\frac{1}{2^{n}}\right) \cdot\left[\frac{1}{1^{n}}+\frac{1}{3^{n}}+\frac{1}{5^{n}}+\frac{1}{7^{n}}+\cdots\right]+\frac{1}{2^{n}} \cdot \frac{1}{2^{n}}\left[\frac{1}{1^{n}}+\frac{1}{2^{n}}+\frac{1}{3^{n}}+\frac{1}{4^{n}}+\cdots\right]
\end{aligned}
$$




$$
=\left(1+\frac{1}{2^{n}}+\frac{1}{\left(2^{n}\right)^{2}}+\frac{1}{\left(2^{n}\right)^{3}}+\cdots\right) \cdot\left[\frac{1}{1^{n}}+\frac{1}{3^{n}}+\frac{1}{5^{n}}+\frac{1}{7^{n}}+\cdots\right]
$$

Note that the first series on the right side is a geometric series with common ratio $\frac{1}{2^{n}}$.

$$
\Rightarrow \zeta(n)=\frac{2^{n}}{2^{n}-1} \cdot\left[\frac{1}{1}+\frac{1}{3^{n}}+\frac{1}{5^{n}}+\frac{1}{7^{n}}+\cdots\right]=\frac{2^{n}}{2^{n}-1} \sum_{j=0}^{\infty} \frac{1}{(2 j+1)^{n}}
$$

\section{Corollary $\mathbf{1 . 2 .}$}

$$
\zeta(2 n)=\frac{2^{2 n}}{2^{2 n}-1} a_{0} \pi^{2 n}
$$

Replacing $n$ by $2 n$ in Lemma 1.2., and using the first part of Corollary 1.1., one can prove Corollary 1.2.

Using Corollary 1.2., one can compute the values of Riemann's zeta function at even positive integers as illustrated in the following examples:

$$
\begin{aligned}
& \zeta(2)=\frac{4}{3} \cdot \frac{\pi^{2}}{8}=\frac{\pi^{2}}{6} . \\
& \zeta(4)=\frac{16}{15} \cdot \frac{\pi^{4}}{96}=\frac{\pi^{4}}{90} . \\
& \zeta(6)=\frac{64}{63} \cdot \frac{1}{960} \pi^{6}=\frac{\pi^{6}}{945} . \\
& \zeta(8)=\frac{256}{255} \cdot \frac{17}{161280} \pi^{8}=\frac{\pi^{8}}{9450} . \\
& \zeta(10)=\frac{1024}{1023} \cdot \frac{31}{2903040} \pi^{10}=\frac{\pi^{10}}{93555} .
\end{aligned}
$$




$$
\begin{aligned}
& \zeta(12)=\frac{2^{12}}{2^{12}-1} \cdot \frac{691}{638668800} \pi^{12}=\frac{691}{638512875} \pi^{12} . \\
& \zeta(14)=\frac{2^{14}}{2^{14}-1} \cdot \frac{5461}{49816166400} \pi^{14}=\frac{10922}{99626251725} \pi^{14} .
\end{aligned}
$$

\section{References}

[1] Blatner D (1997) The Joy of $\Pi$, Walker and Company, New York.

[2] Edwards HM (2001) Riemann's Zeta Function, Dover Publications, Inc.

[3] Euler L (1988) Introduction to Analysis of the Infinite, Vol. 1 (Translation by J. D. Blanton), Springer-Verlag.

[4] Karim MR and C Gonzalez (2010) A Class of Series and Their Sums. Proceedings of NCUR Missoula, Montana.

[5] Markushevich Al (1977) Theory of Functions (3 Volumes in one), Chelsea.

[6] Nahin PJ (2006) Dr. Euler's Fabulous Formula, Princeton University Press.

[7] Saff EB and AD Snider (2003) Fundamentals of Complex Analysis with Applications to Engineering and Science. $3^{\text {rd }}$ Edition, Prentice Hall.

[8] Stewart J (2008) Calculus - Early Transcendentals, 6 th Edition, Thomson. 